\title{
Inertial Manifolds for 2D Generalized MHD System
}

\section{Zhaoqin Yuan, Liang Guo, Guoguang Lin*}

Department of Mathematics, Yunnan University, Kunming, China Email: 15925159599@163.com, ${ }^{*}$ yuanzq091@163.com, ${ }^{*}$ gglin@ynu.edu.cn.

Received 5 June 2015; accepted 16 August 2015; published 20 August 2015

Copyright $(\underset{2015}{ }$ by authors and Scientific Research Publishing Inc.

This work is licensed under the Creative Commons Attribution International License (CC BY). http://creativecommons.org/licenses/by/4.0/

(c) (i) Open Access

\section{Abstract}

In this paper, we prove the existence of inertial manifolds for 2D generalized MHD system under the spectral gap condition.

\section{Keywords}

\section{MHD System, Spectral Gap, Inertial Manifolds}

\section{Introduction}

In [1], Yuan, Guo and Lin prove the existence of global attractors and dimension estimation of a 2D generalized magnetohydrodynamic (MHD) system:

$$
\left\{\begin{array}{l}
\frac{\partial u}{\partial t}+(u \cdot \nabla) u-(v \cdot \nabla) v+\gamma(-\Delta)^{2 \alpha} u=f(x) \\
\frac{\partial v}{\partial t}+(u \cdot \nabla) v-(v \cdot \nabla) u+\eta(-\Delta)^{2 \beta} v=g(x) \\
\nabla u=\nabla v=0 \\
(u, v)(x, 0)=\left(u_{0}, v_{0}\right)(x) \\
\left.u(x, t)\right|_{\partial \Omega}=\left.v(x, t)\right|_{\partial \Omega}=0 .
\end{array}\right.
$$

where $u$ is the fluid velocity field, $v$ is the magnetic field, $\gamma$ is the constant kinematic viscosity and $\eta$ is constant magnetic diffusivity. $\Omega \subset R^{n}$ is a bounded domain with a sufficiently smooth boundary $\partial \Omega, \gamma, \eta>0, \alpha, \beta>\frac{n}{2}$.

${ }^{*}$ Corresponding author. 
More results about inertial manifolds can be founded in [2]-[11].

In this paper, we consider the following 2D generalized MHD system:

$$
\left\{\begin{array}{l}
\frac{\partial u}{\partial t}+(u \cdot \nabla) u-(v \cdot \nabla) v+\gamma(-\Delta)^{2 \alpha} u=f(x) \\
\frac{\partial v}{\partial t}+(u \cdot \nabla) v-(v \cdot \nabla) u+\gamma(-\Delta)^{2 \alpha} v=g(x) \\
\nabla u=\nabla v=0 \\
(u, v)(x, 0)=\left(u_{0}, v_{0}\right)(x) \\
\left.u(x, t)\right|_{\partial \Omega}=\left.v(x, t)\right|_{\partial \Omega}=0 .
\end{array}\right.
$$

where $u$ is the fluid velocity field, $v$ is the magnetic field, $\gamma$ is the constant kinematic viscosity and $\eta$ is the constant magnetic diffusivity. $\Omega \subset R^{n}$ is a bounded domain with a sufficiently smooth boundary $\partial \Omega$, $\gamma>0, \alpha>\frac{n}{2}$.

This paper is organized as follows. In Section 2, we introduce basic concepts concerning inertial manifolds. In Section 3, we obtain the existence of the inertial manifolds.

\section{Preliminaries}

We rewrite the problem (1.2) as a first order differential equation, the problem (1.2) is equivalent to:

$$
\left\{\begin{array}{l}
U_{t}+A U=F(U), \quad t>0 \\
U(0)=U_{0}
\end{array}\right.
$$

where $U=\left(\begin{array}{l}u \\ v\end{array}\right), U_{t}=\left(\begin{array}{l}u_{t} \\ v_{t}\end{array}\right)$, and

$$
A=\left(\begin{array}{cc}
\gamma(-\Delta)^{2 \alpha} & 0 \\
0 & \gamma(-\Delta)^{2 \alpha}
\end{array}\right), F(U)=\left(\begin{array}{c}
f(x)-(u \cdot \nabla) u+(v \cdot \nabla) v \\
g(x)-(u \cdot \nabla) v+(v \cdot \nabla) u
\end{array}\right)
$$

Let $H$ is a Banach space, $H=L^{2}(\Omega) \times L^{2}(\Omega),\|\cdot\|$ is norm of $H,(\cdot, \cdot)$ is inner product of $H$, $\|U\|^{2}=\|u\|^{2}+\|v\|^{2} ; V_{1}=D\left((-\Delta)^{\alpha}\right) \times D\left((-\Delta)^{\alpha}\right)$, for any solution $U \in V_{1}$ of the problem (2.1), $\|U\|_{V_{1}}=\left(\left\|(-\Delta)^{\alpha} u\right\|^{2}+\left\|(-\Delta)^{\alpha} v\right\|^{2}\right)^{\frac{1}{2}},\|\cdot\|_{V_{1}}$ is norm of $V_{1}$.

Definition 2.1. Suppose $S(t)$ denote the semi-group of solutions to the problem (2.l) in $V_{1} \times[0, T](T>0)$, subset $M$ is an inertial manifolds of the problem (2.l), that is $M$ satisfying the following properties:

1. $M$ is a finite dimensional Lipshitz manifold;

2. $M$ is positively invariant under $S(t)$, that is, $S(t) M \subset M$ for all $t \geq 0$;

3. $M$ is attracts every trajectory exponentially, i.e., for every $U_{0} \in V_{1}$,

$$
\operatorname{dist}\left(S(t) U_{0}, M\right) \rightarrow 0, t \rightarrow+\infty .
$$

We now recall some notions. Let $A$ is a closed linear operator on $H$ satisfying the following Standing Hypothesis 2.2.

Standing Hypothesis 2.2. We suppose that $A$ is a positive definite, self-adjoint operator with a discrete spectrum, $A^{-1}$ compacts in $H$. Assume $w_{j}=\left(\begin{array}{l}u_{j} \\ v_{j}\end{array}\right)$ is the orthonormal basis in $H$ consisting of the corresponding eigenfunctions of the operator $A$. Say

$$
A w_{j}=\lambda_{j} w_{j}, j=1,2, \cdots,
$$


$0<\lambda_{1} \leq \lambda_{2} \leq \cdots$, each with finite multiplicity and $\lim _{j \rightarrow+\infty} \lambda_{j}=+\infty$.

Let now $\lambda_{N}$ and $\lambda_{N+1}$ be two successive and different eigenvalues with $\lambda_{N}<\lambda_{N+1}$, let further $P$ be the orthogonal projection onto the first $N$ eigenvectors of the operator $A$.

Let the bound absorbing set $B_{\rho} \subseteq V_{1}$, we define a smooth truncated function by setting $\theta: R^{+} \rightarrow[0,1]$ is defined as

$$
\left\{\begin{array}{l}
\theta(\xi)=1, \quad 0 \leq \xi \leq 1, \\
\theta(\xi)=0, \quad \xi \geq 2 \\
\left|\theta^{\prime}(\xi)\right| \leq 2, \quad \xi \geq 0 \\
\theta_{\rho}(r)=\theta\left(\frac{r}{\rho}\right)
\end{array}\right.
$$

Suppose that $F_{\theta}(U)=\theta_{\rho}\left(\left|A^{\frac{1}{2}} U\right|\right) F(U)$, the problem (2.1) is equivalent to the following preliminary equation:

$$
\left\{\begin{array}{l}
\frac{\mathrm{d} U}{\mathrm{~d} t}+A U=F_{\theta}(U), t>0 \\
U(0)=U_{0}
\end{array}\right.
$$

Denote by $P_{N}$ is the orthogonal projection of $H$ onto $H:=\operatorname{span}\left\{w_{1}, \cdots, w_{N}\right\}$, and $Q_{N}=I-P_{N}$. Set $p=P_{N} U, q=Q_{N} U$, then Equation (2.4) is equivalent to

$$
\begin{aligned}
& \frac{\mathrm{d} p}{\mathrm{~d} t}+A p=P_{N} F_{\theta}(p+q), \\
& \frac{\mathrm{d} q}{\mathrm{~d} t}+A q=Q_{N} F_{\theta}(p+q) .
\end{aligned}
$$

Lemma 2.3. Defined by $F(U)$ of the problem (2.1) on the bounded set of $V_{1}$ is a Lipschitz function, for every $U_{1}=\left(\begin{array}{l}u_{1} \\ v_{1}\end{array}\right), U_{2}=\left(\begin{array}{l}u_{2} \\ v_{2}\end{array}\right) \in V_{1}$, there exist a constant $C>0$ such that

$$
\left\|F\left(U_{1}\right)-F\left(U_{2}\right)\right\| \leq C\left\|A^{\frac{1}{2}}\left(U_{1}-U_{2}\right)\right\|
$$

where $C=C_{3} k$.

Proof. Assume $U_{1}, U_{2} \in V_{1}$, and let $U=U_{1}-U_{2}=\left(\begin{array}{l}u \\ v\end{array}\right)$, use the fact that $\|U\|_{V_{1}} \leq M_{1}$ and using Poincare inequality $\|U\| \leq k\left\|A^{1 / 2} U\right\|$, we have

$$
\begin{aligned}
& \left|\left(F\left(U_{1}\right)-F\left(U_{2}\right), U\right)\right| \\
& \leq\left|\left(-u_{1} \nabla u_{1}+u_{2} \nabla u_{2}+v_{1} \nabla v_{1}-v_{2} \nabla v_{2}, u\right)\right|+\left|\left(-u_{1} \nabla v_{1}+u_{2} \nabla v_{2}+v_{1} \nabla u_{1}-v_{2} \nabla u_{2}, v\right)\right| \\
& \leq C_{0} M_{1}\|u\|^{2}+C_{1} M_{1}\|u\|\|v\|+C_{2} M_{1}\|v\|^{2} \leq\left(C_{0} M_{1}+C_{1} M_{1}\right)\|u\|^{2}+\left(C_{1} M_{1}+C_{2} M_{1}\right)\|v\|^{2} \\
& \leq C_{3}\left(\|u\|^{2}+\|v\|^{2}\right)=C_{3}\|U\|^{2} \leq C_{3} k\left\|A^{\frac{1}{2}} U\right\|\|U\|=C\left\|A^{\frac{1}{2}} U\right\|\|U\|,
\end{aligned}
$$

where $C_{3}=\max \left\{C_{0} M_{1}+C_{1} M_{1}, C_{1} M_{1}+C_{2} M_{1}\right\}$, so we can get 


$$
\left\|F\left(U_{1}\right)-F\left(U_{2}\right)\right\| \leq C\left\|A^{\frac{1}{2}} U\right\| .
$$

Lemma 2.3 is proved.

Lemma 2.4. Let $T>0$ be fixed, for any $N$ and all $t \in[0, T]$, there exist $\zeta>0$ such that

$$
\left\|Q_{N}\left(U_{1}(t)-U_{2}(t)\right)\right\| \leq \zeta\left\|P_{N}\left(U_{1}(t)-U_{2}(t)\right)\right\|
$$

otherwise, there exist constants $C_{4}=\exp \left(C^{2} T\right)$ and $C_{5}=-\frac{\zeta^{2}}{\zeta^{2}+1} \exp \left(-C^{2} T\right)$ are dependent on $\zeta, M_{1}, T$ such that

$$
\left\|U_{1}(t)-U_{2}(t)\right\| \leq C_{4} \exp \left(-C_{5} \lambda_{N+1} t\right)\left\|U_{1}(0)-U_{2}(0)\right\|
$$

and

$$
\left\|U_{1}(t)-U_{2}(t)\right\| \leq \exp \left(C^{2} t\right)\left\|U_{1}(0)-U_{2}(0)\right\|,
$$

for all $U_{1}=\left(\begin{array}{l}u_{1} \\ v_{1}\end{array}\right), U_{2}=\left(\begin{array}{l}u_{2} \\ v_{2}\end{array}\right) \in V_{1}$.

Proof. Let $U_{1}, U_{2}$ with initial values $U_{1}(0), U_{2}(0) \in V_{1}$, respectively, are two different solutions of the problem (2.1), we have the fact that $\|U\|_{V_{1}} \leq M_{1}, \forall t \in[0, T]$. Put $U(t)=U_{1}(t)-U_{2}(t)$, so we obtain that

$$
\frac{\mathrm{d} U}{\mathrm{~d} t}+A U=F\left(U_{1}\right)-F\left(U_{2}\right)
$$

Putting

$$
p(t)=\frac{\left\|A^{\frac{1}{2}} U(t)\right\|^{2}}{\|U(t)\|^{2}}=\frac{\left(A^{\frac{1}{2}} U, A^{\frac{1}{2}} U\right)}{(U, U)} .
$$

For $t \in[0, T]$, taking the derivative of Equation (2.14) with respect to $t$, we have

$$
\begin{aligned}
\frac{\mathrm{d} p}{\mathrm{~d} t} & =\frac{2}{\|U\|^{4}}\left(\|U\|^{2}\left(A^{\frac{1}{2}} U^{\prime}, A^{\frac{1}{2}} U\right)-\left\|A^{\frac{1}{2}} U\right\|^{2}\left(U^{\prime}, U\right)\right) \\
& =\frac{2}{\|U\|^{2}}\left(\left(U^{\prime}, A U\right)-p(t)\left(U^{\prime}, U\right)\right) .
\end{aligned}
$$

From Equation (2.13) and Equation (2.15), we have

$$
\frac{\mathrm{d} p}{\mathrm{~d} t}=\frac{-2}{\|U\|^{2}}\left(A U-\left(F\left(U_{1}\right)-F\left(U_{2}\right)\right), A U-p(t) U\right) .
$$

We notice that Equation (2.14)

$$
(p U, A U-p U)=p\left(A^{\frac{1}{2}} U, A^{\frac{1}{2}} U\right)-p^{2}(U, U)=0,
$$

so we have

$$
(A U, A U-p(t) U)=(A U-p(t) U, A U-p(t) U)=\|A U-p(t) U\|^{2} .
$$

By Equation (2.16) and Equation (2.17), and use the Cauchy-Schwarz inequality, we obtain 


$$
\begin{aligned}
\frac{\mathrm{d} p}{\mathrm{~d} t}+\frac{2}{\|U\|^{2}}\|A U-p(t) U\|^{2} & =\frac{2}{\|U\|^{2}}\left(\left(F\left(U_{1}\right)-F\left(U_{2}\right)\right), A U-p(t) U\right) \\
& \leq \frac{2}{\|U\|^{2}}\left\|F\left(U_{1}\right)-F\left(U_{2}\right)\right\|\|A U-p(t) U\| \\
& \leq \frac{2}{\|U\|^{2}}\|A U-p(t) U\|^{2}+\frac{\left\|F\left(U_{1}\right)-F\left(U_{2}\right)\right\|^{2}}{\|U\|^{2}} \\
& \leq \frac{2}{\|U\|^{2}}\|A U-p(t) U\|^{2}+\frac{C^{2}\left\|A^{\frac{1}{2}} U\right\|^{2}}{\|U\|^{2}} .
\end{aligned}
$$

Then using Lemma 2.3, we have

$$
\frac{\mathrm{d} p}{\mathrm{~d} t} \leq C^{2} p
$$

For $0<\tau<t<T$, integrating the above inequality over $[\tau, t]$, we obtain

$$
\frac{\left\|A^{\frac{1}{2}} U(t)\right\|^{2}}{\|U(t)\|^{2}} \leq \frac{\left\|A^{\frac{1}{2}} U(\tau)\right\|^{2}}{\|U(\tau)\|^{2}} \exp \left(C^{2}(t-\tau)\right)
$$

where $C$ is given as in Lemma 2.3 .

By multiplying (2.13) by $U$, using Cauchy-Schwarz inequality and Lemma 2.3, we have

$$
\frac{1}{2} \frac{\mathrm{d}}{\mathrm{d} t}\|U\|^{2}+\left\|A^{\frac{1}{2}} U\right\|^{2}=\left(F\left(U_{1}\right)-F\left(U_{2}\right), U\right) \leq\left\|F\left(U_{1}\right)-F\left(U_{2}\right)\right\|\|U\| \leq C\left\|A^{\frac{1}{2}} U\right\|\|U\| .
$$

Using Holder inequality, from Equation (2.20) we have

$$
\frac{\mathrm{d}}{\mathrm{d} t}\|U\|^{2}+\|U\|^{2}\left(\frac{\left\|A^{\frac{1}{2}} U\right\|^{2}}{\|U\|^{2}}-C^{2}\right) \leq 0
$$

In Equation (2.19) setting $\tau=t, t=t_{0}$, we obtain

$$
\frac{\left\|A^{\frac{1}{2}} U(t)\right\|^{2}}{\|U(t)\|^{2}} \geq \frac{\left\|A^{\frac{1}{2}} U\left(t_{0}\right)\right\|^{2}}{\left\|U\left(t_{0}\right)\right\|^{2}} \exp \left(-C^{2}\left(t_{0}-t\right)\right) \geq \varepsilon \exp \left(-C^{2} t_{0}\right),
$$

where

$$
\varepsilon=\frac{\left\|A^{\frac{1}{2}} U\left(t_{0}\right)\right\|^{2}}{\left\|U\left(t_{0}\right)\right\|^{2}} .
$$

By Equation (2.21) and Equation (2.22), we have

$$
\frac{\mathrm{d}}{\mathrm{d} t}\|U\|^{2}+\|U\|^{2}\left(\varepsilon \exp \left(-C^{2} t_{0}\right)-C^{2}\right) \leq 0 .
$$

Integrating Equation (2.24) between 0 and $t_{0}$, we obtain

$$
\left\|U\left(t_{0}\right)\right\|^{2} \leq\|U(0)\|^{2} \exp \left(-\varepsilon t_{0} \exp \left(-C^{2} t_{0}\right)+C^{2} t_{0}\right) .
$$


To complete the proof of Lemma 2.4, we consider the following two cases,

$$
\left\|Q_{N} U\left(t_{0}\right)\right\|>\zeta\left\|P_{N} U\left(t_{0}\right)\right\|
$$

and

$$
\left\|Q_{N} U\left(t_{0}\right)\right\| \leq \zeta\left\|P_{N} U\left(t_{0}\right)\right\|
$$

We only consider Equation (2.26), in this case,

$$
\begin{aligned}
\varepsilon & =\frac{\left\|A^{\frac{1}{2}} U\left(t_{0}\right)\right\|^{2}}{\left\|U\left(t_{0}\right)\right\|^{2}}=\frac{\left\|P_{N} A^{\frac{1}{2}} U\left(t_{0}\right)\right\|^{2}+\left\|Q_{N} A^{\frac{1}{2}} U\left(t_{0}\right)\right\|^{2}}{\left\|P_{N} U\left(t_{0}\right)\right\|^{2}+\left\|Q_{N} U\left(t_{0}\right)\right\|^{2}} \\
& \geq \frac{\left\|Q_{N} A^{\frac{1}{2}} U\left(t_{0}\right)\right\|^{2}}{\left(1+\frac{1}{\zeta^{2}}\right)\left\|Q_{N} U\left(t_{0}\right)\right\|^{2}} \geq \frac{\zeta^{2}}{\zeta^{2}+1} \lambda_{N+1},
\end{aligned}
$$

where $\lambda_{N+1}$ is $N+1$ eigenvector of the operator $A$. By Equation (2.25) and Equation (2.28), we obtain

$$
\begin{aligned}
\left\|U\left(t_{0}\right)\right\|^{2} & \leq\|U(0)\|^{2} \exp \left(-\frac{\zeta^{2}}{\zeta^{2}+1} \lambda_{N+1} t_{0} \exp \left(-C^{2} t_{0}\right)+C^{2} t_{0}\right) \\
& \leq\|U(0)\|^{2} \exp \left(-\frac{\zeta^{2}}{\zeta^{2}+1} \lambda_{N+1} T \exp \left(-C^{2} T\right)+C^{2} T\right),
\end{aligned}
$$

since $t_{0}<T$, in Equation (2.29) setting $t=t_{0}$, which proves Equation (2.11), where $C_{4}=\exp \left(C^{2} T\right)$ and $C_{5}=-\frac{\zeta^{2}}{\zeta^{2}+1} \exp \left(-C^{2} T\right)$. Using again Equation (2.20), we have

$$
\frac{\mathrm{d}}{\mathrm{d} t}\|U\|^{2}+2\left\|A^{\frac{1}{2}} U\right\|^{2} \leq 2 C\left\|A^{\frac{1}{2}} U\right\|\|U\| \leq 2\left\|A^{\frac{1}{2}} U\right\|^{2}+C^{2}\|U\|^{2},
$$

then we obtain

$$
\frac{\mathrm{d}}{\mathrm{d} t}\|U\|^{2} \leq C^{2}\|U\|^{2}
$$

Integrating Equation (2.30) between 0 and $t_{0}$, which proves Equation (2.12). Lemma 2.4 is proved.

\section{Inertial Manifolds}

In this section we will prove the existence of the inertial manifolds for solutions to the problem (2.1). We suppose that $A$ satisfies Standing Hypothesis 2.2 and recall that $P$ is the orthogonal projection onto the first $N$ orthonormal eigenvectors of $A$.

Let constants $b, l>0$ be fixed, we define $F=F_{b, l}^{\frac{1}{2}}$ and denote the collection of all functions $\Phi: P_{N} V_{1} \rightarrow Q_{N} V_{1}$ satisfies

$$
\left\{\begin{array}{l}
\operatorname{supp} \Phi \subset\left\{p \in P_{N} V_{1},\left\|A^{\frac{1}{2}} p\right\| \leq 2 \rho\right\}, \\
\left\|A^{\frac{1}{2}} \Phi(p)\right\| \leq b, \quad \forall p \in P_{N} V_{1}, \\
\left\|A^{\frac{1}{2}}\left(\Phi\left(p_{1}\right)-\Phi\left(p_{2}\right)\right)\right\| \leq l\left\|A^{\frac{1}{2}}\left(p_{1}-p_{2}\right)\right\|, \quad \forall p_{1}, p_{2} \in V_{1} .
\end{array}\right.
$$


Note that

$$
d\left(\Phi_{1}, \Phi_{2}\right)=\sup _{p \in P_{N} V_{1}}\left\|A^{\frac{1}{2}}\left(\Phi_{1}(p)-\Phi_{2}(p)\right)\right\|
$$

is the distance of $F_{1}=F_{b, l}^{\frac{1}{2}}$. So $F$ is completely space.

For every $\Phi \in F_{b, l}^{\overline{2}}$ and the initial data $p_{0} \in P_{N} V_{1}$, the initial value problem

$$
\left\{\begin{array}{l}
\frac{\mathrm{d} p}{\mathrm{~d} t}+A p=P_{N} F_{\theta}(p+\Phi(p)), \\
p(0)=p_{0},
\end{array}\right.
$$

possesses a unique solution $p(t)=p\left(t ; \Phi, p_{0}\right)$.

$$
\frac{\mathrm{d} q}{\mathrm{~d} t}+A q=Q_{N} F_{\theta}(p+\Phi(p))
$$

where $Q_{N} F_{\theta}(p+\Phi(p)) \in L^{\infty}(R \times R ; H)$ and the unique solution $q=q\left(t ; \Phi, p_{0}\right)$ in Equation (3.4) is a successive bounded mapping acts from $R \times R$ into $Q_{N} V_{1}$. Particularly, the function

$$
p_{0} \in P_{N} V_{1} \rightarrow q\left(0 ; \Phi, p_{0}\right) \in Q_{N} V_{1} .
$$

by $\Phi \in F_{b, l}^{\frac{1}{2}}$, note that $T \Phi: p_{0} \rightarrow q\left(0 ; \Phi, p_{0}\right)$, we have

$$
T \Phi\left(p_{0}\right)=\int_{-\infty}^{0} \mathrm{e}^{A \tau} Q_{N} F_{\theta}(p(\tau)+\Phi(p(\tau))) \mathrm{d} \tau=q\left(0 ; \Phi, p_{0}\right)
$$

We need to prove the following two conclusions:

1. For $\lambda_{N}^{\frac{1}{2}}$ and $\lambda_{N+1}^{\frac{1}{2}}-\lambda_{N}^{\frac{1}{2}}$ are sufficiently large, $T: F_{b, l}^{\frac{1}{2}} \rightarrow F_{b, l}^{\frac{1}{2}}$ is a contraction.

2. $\Phi_{0}$ is a unique fixed point in $T, M=\operatorname{Graph}\left(\Phi_{0}\right)$ is a inertial manifold of $2 \mathrm{D}$ generalized MHD system. So we give the following Lemmas.

Lemma 3.1. Let $\forall \Phi \in F_{b, l}^{\frac{1}{2}}$, so we have

$$
\operatorname{supp} \Phi \subset\left\{p \in P_{N} V_{1},\left\|A^{\frac{1}{2}} p\right\| \leq 2 \rho\right\} .
$$

Proof. The proof is similar to Temam [3].

Lemma 3.2. Let $\forall \Phi \in F_{b, l}^{\frac{1}{2}}$, for $U_{i}=p_{i}+\Phi\left(p_{i}\right)(i=1,2)$, there exists constant $M_{2}, M_{3}>0$ such that

$$
\left\|F_{\theta}\left(U_{1}\right)\right\| \leq M_{2}
$$

and

$$
\left\|F_{\theta}\left(U_{1}\right)-F_{\theta}\left(U_{2}\right)\right\| \leq M_{3}(1+l)\left\|A^{\frac{1}{2}}\left(p_{1}-p_{2}\right)\right\|, \quad \forall p_{1}, p_{2} \in P_{N} V_{1} .
$$

Proof. For any $\Phi \in F_{b, l}^{\frac{1}{2}}$, and $p_{1}, p_{2} \in P_{N} V_{1}$, we denote $U_{i}=p_{i}+\Phi\left(p_{i}\right)(i=1,2)$, using Lemma 2.3 and see ([3], Chapter 8: Lemma 2.1 and Lemma 2.2), we derive that there exists constant $M_{2}, M_{3}>0$ such that

$$
\left\|F_{\theta}\left(U_{1}\right)\right\| \leq M_{2}
$$

and 


$$
\left\|F_{\theta}\left(U_{1}\right)-F_{\theta}\left(U_{2}\right)\right\| \leq M_{3}\left\|A^{\frac{1}{2}}\left(U_{1}-U_{2}\right)\right\|,
$$

which proves Equation (3.8). We now prove Equation (3.9), by the definition of $F_{b, l}^{\frac{1}{2}}$, we have

$$
\left\|A^{\frac{1}{2}}\left(\Phi\left(p_{1}\right)-\Phi\left(p_{2}\right)\right)\right\| \leq l\left\|A^{\frac{1}{2}}\left(p_{1}-p_{2}\right)\right\| .
$$

And we have

$$
\left\|A^{\frac{1}{2}}\left(U_{1}-U_{2}\right)\right\| \leq\left\|A^{\frac{1}{2}}\left(p_{1}-p_{2}\right)\right\|+\left\|A^{\frac{1}{2}}\left(\Phi\left(p_{1}\right)-\Phi\left(p_{2}\right)\right)\right\| \leq(1+l)\left\|A^{\frac{1}{2}}\left(p_{1}-p_{2}\right)\right\| .
$$

Substituting Equation (3.13) into Equation (3.11) we obtain Equation (3.9). Lemma 3.2 is proved.

Lemma 3.3. Let $p_{0} \in P_{N} V_{1}$, one has $T \Phi\left(p_{0}\right) \in Q_{N} V_{1}$ and $\left\|A^{\frac{1}{2}}\left(T \Phi\left(p_{0}\right)\right)\right\| \leq b_{1}$, where $b_{1}=6 \mathrm{e}^{-\frac{1}{2}} M_{2} \lambda_{N+1}^{-\frac{1}{2}}$, for $\lambda_{N+1}$ is sufficiently large one has $b_{1}<b$.

Proof. Let $p_{0} \in P_{N} V_{1}$, according to the definition of $T$, we have $T \Phi\left(p_{0}\right) \in Q_{N} V_{1}$, from Equation (3.6) and Equation (3.10), we have

$$
\begin{aligned}
\left\|A^{\frac{1}{2}}\left(T \Phi\left(p_{0}\right)\right)\right\| & \leq \int_{-\infty}^{0}\left\|A^{\frac{1}{2}} \mathrm{e}^{A \tau} Q_{N} F_{\theta}(p(\tau)+\Phi(p(\tau)))\right\| \mathrm{d} \tau \\
& \leq \int_{-\infty}^{0}\left\|\left(A Q_{N}\right)^{\frac{1}{2}} \mathrm{e}^{A \tau}\right\|_{L\left(Q_{N} H\right)}\left\|F_{\theta}(p(\tau)+\Phi(p(\tau)))\right\| \mathrm{d} \tau \\
& \leq M_{2} \int_{-\infty}^{0}\left\|\left(A Q_{N}\right)^{\frac{1}{2}} \mathrm{e}^{A \tau}\right\|_{L\left(Q_{N} H\right)} \mathrm{d} \tau .
\end{aligned}
$$

Let $\delta \in R$ and $\tau<0$, suppose that $K_{2}(\delta)=\delta^{\delta} \mathrm{e}^{-\delta}$ and

$$
K_{3}(\delta)=\left\{\begin{array}{lc}
1, & \delta<0, \\
\mathrm{e}^{-\delta}+\frac{K_{2}(\delta)}{1-\delta} \delta^{1-\delta}, & 0 \leq \delta<1 .
\end{array}\right.
$$

So we obtain

$$
\left\|\left(A Q_{N}\right)^{\delta} \mathrm{e}^{A Q_{N} \tau}\right\|_{L\left(Q_{N} H\right)}= \begin{cases}K_{2}(\delta)|\tau|^{-\delta}, & -\frac{\delta}{\lambda_{N+1}} \leq \tau<0, \\ \lambda_{N+1}^{\delta} \mathrm{e}^{\tau \lambda_{N+1}}, & \tau<-\frac{\delta}{\lambda_{N+1}} .\end{cases}
$$

Further more, for $\delta<1$, we have

$$
\int_{-\infty}^{0}\left\|\left(A Q_{N}\right)^{\delta} \mathrm{e}^{A Q_{N} \tau}\right\|_{L\left(Q_{N} H\right)} \mathrm{d} \tau \leq K_{3}(\delta) \lambda_{N+1}^{\delta-1} .
$$

Setting $\delta=\frac{1}{2}$ in $K_{2}\left(\frac{1}{2}\right), K_{3}\left(\frac{1}{2}\right)$, then substituting $K_{2}\left(\frac{1}{2}\right), K_{3}\left(\frac{1}{2}\right)$ into Equation (3.15) and Equation (3.16), and from Equation (3.14) we can derive that

$$
\left\|A^{\frac{1}{2}}\left(T \Phi\left(p_{0}\right)\right)\right\| \leq 3 K_{3}\left(\frac{1}{2}\right) \lambda_{N+1}^{-\frac{1}{2}} M_{2} \leq 6 \lambda_{N+1}^{-\frac{1}{2}} M_{2} \mathrm{e}^{-\frac{1}{2}} .
$$


Lemma 3.3 is proved.

Lemma 3.4. Let

$$
\mu_{N}=\left(\lambda_{N+1}-\lambda_{N}\right)-M_{3}(1+l) \lambda_{N}^{\frac{1}{2}}>0,
$$

so for every $\Phi \in F_{b, l}^{\frac{1}{2}}$, one has

$$
\left\|A^{\frac{1}{2}}\left(T \Phi\left(p_{01}\right)-T \Phi\left(p_{02}\right)\right)\right\| \leq l_{1}\left\|A^{\frac{1}{2}}\left(p_{01}-p_{02}\right)\right\|, \quad \forall p_{01}, p_{02} \in P_{N} V_{1},
$$

here

$$
\begin{gathered}
l_{1}=M_{3}(1+l) \lambda_{N+1}^{-\frac{1}{2}}\left[\frac{1}{\sqrt{2}}+\left(1-\zeta_{N} \xi_{N}\right)^{-1}\right] \mathrm{e}^{-\frac{1}{2}} \exp \left(\frac{\zeta_{N} \xi_{N}}{2}\right), \\
\zeta_{N}=\frac{\lambda_{N}}{\lambda_{N+1}}, \\
\xi_{N}=1+M_{3}(1+l) \lambda_{N}^{-\frac{1}{2}} .
\end{gathered}
$$

Proof. For any given $\Phi \in F_{b, l}^{\frac{1}{2}}$, let $p_{1}=p_{1}(t), p_{2}=p_{2}(t)$ are the solutions of the following initial value problem,

$$
\left\{\begin{array}{l}
\frac{\mathrm{d} p_{1}}{\mathrm{~d} t}+A p_{1}=P_{N} F_{\theta}\left(U_{1}\right) \\
p_{1}(0)=p_{01}
\end{array}\right.
$$

and

$$
\left\{\begin{array}{l}
\frac{\mathrm{d} p_{2}}{\mathrm{~d} t}+A p_{2}=P_{N} F_{\theta}\left(U_{2}\right), \\
p_{2}(0)=p_{02}
\end{array}\right.
$$

here $U_{i}=p_{i}+\Phi\left(p_{i}\right), i=1,2$. Suppose that $p(t)=p_{1}(t)-p_{2}(t)$, so we have

$$
\left\{\begin{array}{l}
\frac{\mathrm{d} p}{\mathrm{~d} t}+A p=P_{N}\left(F_{\theta}\left(U_{1}\right)-F_{\theta}\left(U_{2}\right)\right) \\
p(0)=p_{01}-p_{02}
\end{array}\right.
$$

Multiplying the first equation in Equation (3.25) by $A p$, using Equation (3.9) in Lemma 3.2, we obtain

$$
\frac{1}{2} \frac{\mathrm{d}}{\mathrm{d} t}\left\|A^{\frac{1}{2}} p\right\|^{2}+\|A p\|^{2} \geq-\left\|F_{\theta}\left(U_{1}\right)-F_{\theta}\left(U_{2}\right)\right\|\|A p\| \geq-M_{3}(1+l)\left\|A^{\frac{1}{2}} p\right\|\|A p\| .
$$

So we have

$$
\frac{\mathrm{d}}{\mathrm{d} t}\left\|A^{\frac{1}{2}} p\right\|+\left(\lambda_{N}+M_{3}(1+l) \lambda_{N}^{\frac{1}{2}}\right)\left\|A^{\frac{1}{2}} p\right\| \geq 0 .
$$

For $t \leq 0$, from Equation (3.27) we have

$$
\left\|A^{\frac{1}{2}} p(t)\right\| \leq\left\|A^{\frac{1}{2}} p(0)\right\| \exp \left[-t\left(\lambda_{N}+M_{3}(1+l) \lambda_{N}^{\frac{1}{2}}\right)\right] .
$$

By Lemma 2.3, to do the following estimate,using Equation (3.11) and Equation (3.28) we obtain 


$$
\begin{aligned}
& \left\|A^{\frac{1}{2}}\left(T \Phi\left(p_{01}\right)-T \Phi\left(p_{02}\right)\right)\right\| \leq \int_{-\infty}^{0}\left\|A^{\frac{1}{2}} \mathrm{e}^{A t} Q_{N}\left(F_{\theta}\left(U_{1}\right)-F_{\theta}\left(U_{2}\right)\right)\right\| \mathrm{d} t \\
& \leq \int_{-\infty}^{0}\left\|\left(A Q_{N}\right)^{\frac{1}{2}} \mathrm{e}^{A t}\right\|_{L\left(Q_{N} H\right)}\left\|F_{\theta}\left(U_{1}\right)-F_{\theta}\left(U_{2}\right)\right\| \mathrm{d} t \\
& \leq M_{3}(1+l) \int_{-\infty}^{0}\left\|\left(A Q_{N}\right)^{\frac{1}{2}} \mathrm{e}^{A t}\right\|_{L\left(Q_{N} H\right)}\left\|A^{\frac{1}{2}} p\right\| \mathrm{d} t \\
& \leq M_{3}(1+l)\left\|A^{\frac{1}{2}} p(0)\right\| \int_{-\infty}^{0}\left\|\left(A Q_{N}\right)^{\frac{1}{2}} \mathrm{e}^{A t}\right\|_{L\left(Q_{N} H\right)} \mathrm{e}^{-\lambda_{N} \xi_{N} t} \mathrm{~d} t
\end{aligned}
$$

here $\xi_{N}=1+M_{3}(1+l) \lambda_{N}^{-\frac{1}{2}}$. From Equation (3.15), we have



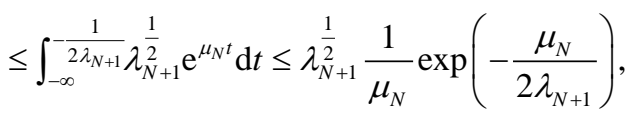

here $\mu_{N}=\lambda_{N+1}-\lambda_{N} \xi_{N}=\lambda_{N+1}\left(1-\zeta_{N} \xi_{N}\right), \zeta_{N}=\frac{\lambda_{N}}{\lambda_{N+1}}$.

Hence,

$$
\int_{-\infty}^{-\frac{1}{2 \lambda_{N+1}} \|}\left\|\left(A Q_{N}\right)^{\frac{1}{2}} \mathrm{e}^{A t}\right\|_{L\left(Q_{N} H\right)} \mathrm{e}^{-\lambda_{N} \xi_{N} t} \mathrm{~d} t \leq \lambda_{N+1}^{-\frac{1}{2}} \mathrm{e}^{-\frac{1}{2}}\left(1-\zeta_{N} \xi_{N}\right)^{-1} \exp \left(\frac{\zeta_{N} \xi_{N}}{2}\right) .
$$

Then from Equation (3.15) we have

$$
\begin{aligned}
& \int_{-\frac{1}{2 \lambda_{N+1}}}^{0}\left\|\left(A Q_{N}\right)^{\frac{1}{2}} \mathrm{e}^{A t}\right\|_{L\left(Q_{N} H\right)} \mathrm{e}^{-\lambda_{N} \xi_{N} t} \mathrm{~d} t \leq \int_{-\frac{1}{2 \lambda_{N+1}}}^{0} K_{2}\left(\frac{1}{2}\right)|t|^{-\frac{1}{2}} \mathrm{e}^{-\lambda_{N} \xi_{N} t} \mathrm{~d} t \\
& \leq(2 \mathrm{e})^{-\frac{1}{2}} \exp \left(\frac{\lambda_{N} \zeta_{N}}{2 \lambda_{N+1}}\right) \int_{-\frac{1}{2 \lambda_{N+1}}}^{0}|t|^{-\frac{1}{2}} \mathrm{~d} t \leq(2 \mathrm{e})^{-\frac{1}{2}} \lambda_{N+1}^{-\frac{1}{2}} \exp \left(\frac{\zeta_{N} \xi_{N}}{2}\right) .
\end{aligned}
$$

Combining Equation (3.31) and Equation (3.32), we obtain

$$
\int_{-\infty}^{0}\left\|\left(A Q_{N}\right)^{\frac{1}{2}} \mathrm{e}^{A t}\right\|_{L\left(Q_{N} H\right)} \mathrm{e}^{-\lambda_{N} \xi_{N} t} \mathrm{~d} t \leq \lambda_{N+1}^{-\frac{1}{2}} \mathrm{e}^{-\frac{1}{2}}\left[\left(1-\zeta_{N} \xi_{N}\right)^{-1}+2^{-\frac{1}{2}}\right] \exp \left(\frac{\zeta_{N} \xi_{N}}{2}\right) .
$$

Substituting Equation (3.33) into Equation (3.29), we obtain

$$
\left\|A^{\frac{1}{2}}\left(T \Phi\left(p_{01}\right)-T \Phi\left(p_{02}\right)\right)\right\| \leq l_{1}\left\|A^{\frac{1}{2}}\left(p_{01}-p_{02}\right)\right\| .
$$

Lemma 3.4 is proved.

Lemma 3.5. Let $\mu_{N}>0$ is defined as in Lemma 3.4, for all $\Phi_{1}, \Phi_{2} \in F_{b, l}^{\frac{1}{2}}$,

$$
\left\|A^{\frac{1}{2}}\left(T \Phi_{1}\left(p_{0}\right)-T \Phi_{2}\left(p_{0}\right)\right)\right\| \leq K_{0} d\left(\Phi_{1}, \Phi_{2}\right), \forall p_{0} \in P_{N} V_{1},
$$

here $K_{0}=M_{3}\left(6 \lambda_{N+1}^{-\frac{1}{2}} \mathrm{e}^{-\frac{1}{2}}+\lambda_{N}^{-\frac{1}{2}} l_{1}\right), \quad l_{1}$ is defined by Equation (3.20), $d\left(\Phi_{1}, \Phi_{2}\right)$ is defined by Equation (3.2). Proof. Let $p_{i}=p_{i}\left(t ; \Phi_{i}, p_{0}\right), U_{i}=p_{i}+\Phi_{i}\left(p_{i}\right), i=1,2$, and let $p=p_{1}-p_{2}$ is the solution of the initial 
value problem (3.25), then by the same way as in Lemma 3.2 we can prove that

$$
\begin{aligned}
& \left\|F_{\theta}\left(U_{1}\right)-F_{\theta}\left(U_{2}\right)\right\| \leq M_{3}\left\|A^{\frac{1}{2}}\left(U_{1}-U_{2}\right)\right\| \\
& \leq M_{3}\left(\left\|A^{\frac{1}{2}}\left(p_{1}-p_{2}\right)\right\|+\left\|A^{\frac{1}{2}}\left(\Phi_{1}\left(p_{1}\right)-\Phi_{2}\left(p_{2}\right)\right)\right\|\right) \\
& \leq M_{3}\left\|A^{\frac{1}{2}}\left(p_{1}-p_{2}\right)\right\|+M_{3}\left(\left\|A^{\frac{1}{2}}\left(\Phi_{1}\left(p_{1}\right)-\Phi_{1}\left(p_{2}\right)\right)\right\|+\left\|A^{\frac{1}{2}}\left(\Phi_{1}\left(p_{2}\right)-\Phi_{2}\left(p_{2}\right)\right)\right\|\right) \\
& \leq M_{3}\left[(1+l)\left\|A^{\frac{1}{2}}\left(p_{1}-p_{2}\right)\right\|+d\left(\Phi_{1}, \Phi_{2}\right)\right] .
\end{aligned}
$$

From the first inequality of Equation (3.26) and the following estimate, we have

$$
\|A p\|=\left\|A^{\frac{1}{2}} A^{\frac{1}{2}} p\right\| \leq \lambda_{N}^{\frac{1}{2}}\left\|A^{\frac{1}{2}} p\right\|,
$$

then from the last inequality of Equation (3.35), we obtain

$$
\frac{1}{2} \frac{\mathrm{d}}{\mathrm{d} t}\left\|A^{\frac{1}{2}} p\right\|^{2}+\lambda_{N}\left\|A^{\frac{1}{2}} p\right\|^{2} \geq-M_{3}(1+l) \lambda_{N}^{\frac{1}{2}}\left\|A^{\frac{1}{2}} p\right\|^{2}-M_{3} \lambda_{N}^{\frac{1}{2}} d\left(\Phi_{1}, \Phi_{2}\right)\left\|A^{\frac{1}{2}} p\right\| .
$$

From Equation (3.36), we have

$$
\frac{\mathrm{d}}{\mathrm{d} t}\left\|A^{\frac{1}{2}} p\right\|+\left(\lambda_{N}+M_{3}(1+l) \lambda_{N}^{\frac{1}{2}}\right)\left\|A^{\frac{1}{2}} p\right\| \geq-M_{3} \lambda_{N}^{\frac{1}{2}} d\left(\Phi_{1}, \Phi_{2}\right) .
$$

Due to $p(0)=0$, integrating Equation (3.37) over $[0, t<0]$, we have

$$
\left\|A^{\frac{1}{2}} p\right\| \leq M_{3} \lambda_{N}^{\frac{1}{2}}\left(\lambda_{N} \xi_{N}\right)^{-1}\left(\exp \left(-t \lambda_{N} \xi_{N}\right)-1\right) \mathrm{d}\left(\Phi_{1}, \Phi_{2}\right) .
$$

From Equation (3.6), Equation (3.35) and Equation (3.38), we have

$$
\begin{aligned}
& \left\|A^{\frac{1}{2}}\left(T \Phi_{1}\left(p_{0}\right)-T \Phi_{2}\left(p_{0}\right)\right)\right\| \leq \int_{-\infty}^{0}\left\|A^{\frac{1}{2}} \mathrm{e}^{A t} Q_{N}\left(F_{\theta}\left(U_{1}\right)-F_{\theta}\left(U_{2}\right)\right)\right\| \mathrm{d} t \\
& \leq \int_{-\infty}^{0}\left\|\left(A Q_{N}\right)^{\frac{1}{2}} \mathrm{e}^{A t}\right\|_{L(H)}\left\|F_{\theta}\left(U_{1}\right)-F_{\theta}\left(U_{2}\right)\right\| \mathrm{d} t \\
& \leq M_{3} \int_{-\infty}^{0}\left\|\left(A Q_{N}\right)^{\frac{1}{2}} \mathrm{e}^{A t}\right\|_{L(H)}\left[(1+l)\left\|A^{\frac{1}{2}}\left(p_{1}-p_{2}\right)\right\|+d\left(\Phi_{1}, \Phi_{2}\right)\right] \mathrm{d} t \\
& \leq M_{3} d\left(\Phi_{1}, \Phi_{2}\right) \int_{-\infty}^{0}\left\|\left(A Q_{N}\right)^{\frac{1}{2}} \mathrm{e}^{A t}\right\|_{L(H)}\left[1+(1+l) M_{3} \lambda_{N}^{-\frac{1}{2}} \mathrm{e}^{-t \lambda_{N} \xi_{N}}\right] \mathrm{d} t .
\end{aligned}
$$

Then using Equation (3.16), Equation (3.33) and $\mu_{N}>0$, we have

$$
\begin{aligned}
& \left\|A^{\frac{1}{2}}\left(T \Phi_{1}\left(p_{0}\right)-T \Phi_{2}\left(p_{0}\right)\right)\right\| \\
& \leq M_{3}\left[6 \lambda_{N+1}^{-\frac{1}{2}} \mathrm{e}^{-\frac{1}{2}}+M_{3}(1+l) \lambda_{N+1}^{-\frac{1}{2}} \lambda_{N}^{-\frac{1}{2}}\left(\frac{1}{\sqrt{2}}+\left(1-\zeta_{N} \xi_{N}\right)^{-1}\right)\right] d\left(\Phi_{1}, \Phi_{2}\right) \\
& =M_{3}\left(6 \lambda_{N+1}^{-\frac{1}{2}} e^{-\frac{1}{2}}+\lambda_{N}^{-\frac{1}{2}} l_{1}\right) d\left(\Phi_{1}, \Phi_{2}\right)=K_{0} d\left(\Phi_{1}, \Phi_{2}\right) .
\end{aligned}
$$


Lemma 3.5 is proved.

Lemma 3.6. Suppose that $0<l<1$,

$$
\begin{gathered}
\lambda_{N+1}^{\frac{1}{2}}-\lambda_{N}^{\frac{1}{2}} \geq K_{1}, \\
\lambda_{N}^{\frac{1}{2}} \geq K_{2},
\end{gathered}
$$

we have $\mu_{N}>0, l_{1}<l$ and $K_{0}<\frac{1}{2}$, where $K_{0}$ is defined as in Lemma 3.5,

$$
K_{1}=2 M_{3}(1+l) l^{-1}, K_{2}=2 M_{3}\left(6 \mathrm{e}^{-\frac{1}{2}}+l\right) .
$$

Proof. From $\mu_{N}=\left(\lambda_{N+1}-\lambda_{N}\right)-M_{3}(1+l) \lambda_{N}^{\frac{1}{2}}>0$ is equivalent to

$$
1-\zeta_{N} \xi_{N}>0 \text {, }
$$

where $\zeta_{N}$ and $\xi_{N}$ are defined as in Lemma 3.4. To find a sufficient condition of Equation (3.44), suppose that Equation (3.44) hold, so we have

$$
\begin{aligned}
l_{1} & =M_{3}(1+l) \lambda_{N+1}^{-\frac{1}{2}} \mathrm{e}^{-\frac{1}{2}} \mathrm{e}^{\frac{\zeta_{N} \xi_{N}}{2}}\left[\frac{1}{\sqrt{2}}+\left(1-\zeta_{N} \xi_{N}\right)^{-1}\right] \\
& \leq M_{3}(1+l) \lambda_{N+1}^{-\frac{1}{2}}\left[\frac{1}{\sqrt{2}}+\left(1-\zeta_{N} \xi_{N}\right)^{-1}\right] .
\end{aligned}
$$

To make $l_{1}<l$, if and only if it satisfies

$$
\begin{gathered}
M_{3}(1+l) \lambda_{N+1}^{-\frac{1}{2}} \leq \frac{l}{2}, \\
M_{3}(1+l) \lambda_{N+1}^{-\frac{1}{2}} \leq \frac{l}{2}\left(1-\zeta_{N} \xi_{N}\right) .
\end{gathered}
$$

Equation (3.46) is equivalent to

$$
K_{1} \leq \lambda_{N+1}^{\frac{1}{2}}, K_{1}=2 M_{3}(1+l) l^{-1},
$$

If Equation (3.48) is satisfied, so Equation (3.47) is equivalent to $K_{1} \lambda_{N+1}^{-\frac{1}{2}} \leq 1-\zeta_{N} \xi_{N}$ or is equivalent to

$$
K_{1} \lambda_{N+1}^{-\frac{1}{2}}-1+\zeta_{N}+M_{2}(1+l) \lambda_{N+1}^{-\frac{1}{2}} \lambda_{N}^{\frac{1}{2}} \leq 0 .
$$

Suppose that Equation (3.41) is equivalent to

$$
K_{1} \lambda_{N+1}^{-\frac{1}{2}}+\zeta_{N}^{\frac{1}{2}} \leq 1
$$

Hence,

$$
K_{1} \lambda_{N+1}^{-\frac{1}{2}} \zeta_{N}^{\frac{1}{2}}+\zeta_{N} \leq \zeta_{N}^{\frac{1}{2}}
$$

Hence,

$$
K_{1} \lambda_{N+1}^{-\frac{1}{2}}-1+\zeta_{N}+M_{3}(1+l) \lambda_{N+1}^{-\frac{1}{2}} \lambda_{N}^{\frac{1}{2}} \leq K_{1} \lambda_{N+1}^{-\frac{1}{2}}+\zeta_{N}^{\frac{1}{2}}-1 \leq 0 .
$$

Therefore Equation (3.49) follows from Equation (3.52). From Equation (3.41) we conclude that $\mu_{N}>0$, 
Equation (3.48) follows from Equation (3.41), Equation (3.46) follows from Equation (3.48), Equation (3.46) follows from Equation (3.49), and from Equation (3.46) and Equation (3.47) we have $l_{1}<l$. The last we need to prove is $K_{0}<\frac{1}{2}$, from Lemma 3.5, we obtain

$$
K_{0}=M_{3}\left(6 \lambda_{N+1}^{-\frac{1}{2}} \mathrm{e}^{-\frac{1}{2}}+\lambda_{N}^{-\frac{1}{2}} l_{1}\right)<\frac{1}{2},
$$

we notice that $l_{1}<l, \lambda_{N+1}^{\frac{1}{2}} \geq \lambda_{N}^{\frac{1}{2}}, K_{0}<M_{3}\left(6 \mathrm{e}^{-\frac{1}{2}}+l\right) \lambda_{N}^{-\frac{1}{2}}<\frac{1}{2}$. Lemma 3.6 is proved.

From Lemma 3.1 to Lemma 3.6,we can obtain the following conclusions.

Theorem 3.1. Suppose that $F_{b, l}^{\frac{1}{2}}(b>0, l>0)$ is Lipschitz mapping space. $\Phi \in F_{b, l}^{\frac{1}{2}}, \quad \Phi: P_{N} V_{1} \rightarrow Q_{N} V_{1}$ satisfy Equation (3.1) and Equation (3.2), $p_{0} \in P_{N} V_{1}$ and $q\left(0 ; \Phi, p_{0}\right) \in Q_{N} V_{1}$ is the unique solution of Equation (3.3) and Equation (3.4) for $t=0$, respectively. Hence the transformation $T: F_{b, l}^{\frac{1}{2}} \rightarrow F_{b, l}^{\frac{1}{2}}$ is a contraction, and $T$ exists a unique fixed point $\Phi_{0} \in F_{b, l}^{\frac{1}{2}}, M=\operatorname{Graph}\left(\Phi_{0}\right)$ is inertial manifolds of the problem (2.1).

Theorem 3.2. Suppose that $M=\operatorname{Graph}\left(\Phi_{0}\right)$ is the mapping of $\Phi_{0}$, for any $U_{0} \in V_{1}$, there exists $t_{0}>0$ such that, for $t \geq t_{0}$,

$$
\operatorname{dist}\left(S(t) U_{0}, M\right) \leq \operatorname{dist}\left(U_{0}, M\right) \exp \left(-\frac{\ln 2}{2 t_{0}} t\right),
$$

where $t_{0}=\min \left\{\frac{\ln 2}{C^{2}}, \frac{T}{2}\right\}, C$ is defined as in Lemma 2.3.

Proof. Let $U_{1}, U_{2}$ with initial value $U_{1}(0), U_{2}(0) \in V_{1}$, respectively, be two solutions of the problem (2.1). For any arbitrary $N$ and for $t \in[0, T]$, and use the fact $\left\|U_{1}\right\|_{V_{1}} \leq M_{1},\left\|U_{2}\right\|_{V_{1}} \leq M_{1}$, there exists a constant $\zeta>0$ such that Equation (2.10) or Equation (2.11) is satisfied. From Equation (2.12), we have

$$
\left\|U_{1}(t)-U_{2}(t)\right\| \leq 2\left\|U_{1}(0)-U_{2}(0)\right\|, \quad t<2 t_{0} .
$$

Assume $\zeta=\frac{1}{8}$, and for $N>N_{0}, \lambda_{N_{0}+1} \geq \frac{\ln \left(2 C_{4}\right)}{C_{5} t_{0}}$, therefore Equation (2.10) and Equation (2.11) can rewrite

$$
\begin{gathered}
\left\|Q_{N}\left(U_{1}(t)-U_{2}(t)\right)\right\| \leq \frac{1}{8}\left\|P_{N}\left(U_{1}(t)-U_{2}(t)\right)\right\|, \\
\left\|U_{1}(t)-U_{2}(t)\right\| \leq \frac{1}{2}\left\|U_{1}(0)-U_{2}(0)\right\|,
\end{gathered}
$$

Let $U_{1}(0), U_{2}(0) \in V_{1}, t_{0} \leq t \leq 2 t_{0}, B_{\rho} \subset V_{1}$ is absorbing set, the orbital solution $U(t)$ satisfies $\left\|A^{\frac{1}{2}} U(t)\right\| \leq \rho, t \in[0,+\infty)$. Let $U_{2}(0)=U_{02} \in M, U_{02}=P_{N} U_{02}+\Phi_{0}\left(P_{N} U_{02}\right)$ such that

$$
\operatorname{dist}\left(U_{0}, M\right)=\left\|U_{1}(0)-U_{2}(0)\right\| \text {. }
$$

Substituting $S\left(t_{1}\right) U_{1}(0)$ and $S\left(t_{1}\right) U_{2}(0)$ into Equation (3.56) and Equation (3.57), we have 


$$
\begin{aligned}
\operatorname{dist}\left(S\left(t_{1}\right) U_{0}, M\right) & =\inf _{U_{1} \in M}\left\|S\left(t_{1}\right) U_{1}(0)-U_{2}\right\| \leq\left\|S\left(t_{1}\right) U_{1}(0)-S\left(t_{1}\right) U_{2}(0)\right\| \\
& \leq \frac{1}{2}\left\|U_{1}(0)-U_{2}(0)\right\|=\frac{1}{2} \operatorname{dist}\left(U_{0}, M\right) .
\end{aligned}
$$

If Equation (3.56) is satisfied, assume $l=\frac{1}{8}, t_{0} \leq t_{1} \leq 2 t_{0}$, so we have the cone property

$$
\begin{aligned}
\operatorname{dist}\left(S\left(t_{1}\right) U_{0}, M\right) & =\inf _{U_{1} \in M}\left\|S\left(t_{1}\right) U_{1}(0)-\left(P_{N} S\left(t_{1}\right) U_{2}(0)+\Phi\left(P_{N} S\left(t_{1}\right) U_{2}(0)\right)\right)\right\| \\
& \leq\left\|Q_{N} S\left(t_{1}\right) U_{1}(0)-\Phi\left(P_{N} S\left(t_{1}\right) U_{2}(0)\right)\right\| \\
& \leq \frac{1}{8}\left\|P_{N}\left(S\left(t_{1}\right) U_{1}(0)-S\left(t_{1}\right) U_{2}(0)\right)\right\| \\
& \leq \frac{1}{2}\left\|U_{1}(0)-U_{2}(0)\right\|=\frac{1}{2} \operatorname{dist}\left(U_{0}, M\right) .
\end{aligned}
$$

In a word, for $t_{0} \leq t_{1} \leq 2 t_{0}$, whenever $\operatorname{dist}\left(S\left(t_{1}\right) U_{0}, M\right) \leq \frac{1}{2} \operatorname{dist}\left(U_{0}, M\right)$. By the properties of semigroups, for $t_{0} \leq t_{1} \leq 2 t_{0}$, we have

$$
\begin{aligned}
\operatorname{dist}\left(S\left(n t_{1}\right) U_{0}, M\right) & \leq\left(\frac{1}{2}\right)^{n} \operatorname{dist}\left(U_{0}, M\right) \leq \exp \left(-\frac{t \ln 2}{t_{1}}\right) \operatorname{dist}\left(U_{0}, M\right) \\
& \leq \exp \left(-\frac{t \ln 2}{2 t_{0}}\right) \operatorname{dist}\left(U_{0}, M\right) \rightarrow 0\left(n \rightarrow \infty, t \geq t_{0}\right) .
\end{aligned}
$$

Theorem 3.2 is proved.

\section{Supported}

This work is supported by the National Natural Sciences Foundation of People’s Republic of China under Grant 11161057.

\section{References}

[1] Yuan, Z.Q., Guo, L. and Lin, G.G. (2015) Global Attractors and Dimension Estimation of the 2D Generalized MHD System with Extra Force. Applied Mathematics, 6, 724-736. http://dx.doi.org/10.4236/am.2015.64068

[2] Lin, G.G. (2009) An Inertial Manifold of the 2D Swift-Hohenberg Equation. Journal of Yunnan University, 31, 334340.

[3] Temam, R. (1988) Infinite Dimensional Dynamical Systems in Mechanics and Physics. Springer, New York. http://dx.doi.org/10.1007/978-1-4684-0313-8

[4] Constantin, P., Foias, C., Nicolaenko, B. and Temam, R. (1989) Integral Manifolds and Inertial Manifolds for Dissipative Partial Differential Equations. Springer, New York. http://dx.doi.org/10.1007/978-1-4612-3506-4

[5] Lin, G.G. (2011) Nonlinear Evolution Equations. Yunnan University, Kunming.

[6] Babin, A.V. and Vishik, M.I. (1992) Attractors of Evolution Equations. North-Holland, Amsterdam.

[7] Chow, S.-N. and Lu, K. (1988) Invariant Manifolds for Flows in Banach Spaces. Journal of Differential Equations, 74, 285-317. http://dx.doi.org/10.1016/0022-0396(88)90007-1

[8] Chueshov, I.D. (1992) Introduction to the Theory of Inertial Manifolds, (Lecture Notes). Kharkov Univ. Press, Kharkov (in Russian).

[9] Chueshov, I.D. (1999) Introduction to the Theory of Infinite-Dimensional Dissipative Systems. Acta, Kharkov (in Russian) (English Translation, 2002, Acta, Kharkov).

[10] Henry, D. (1981) Geometric Theory of Semilinear Parabolic Equations, Lecture Notes in Math. 840. Springer, BerlinHeidelberg and New York.

[11] Leung, A.W. (1989) Systems of Nonlinear Partial Differential Equations: Applications to Biology and Engineering. MIA, Kluwer, Boston. 